Check for updates

Cite this: RSC Adv., 2017, 7, 33829

\title{
Sandwich-structured rGO/PVDF/PU multilayer coatings for anti-corrosion application
}

\author{
Yuan-Kai Xiao, ${ }^{a}$ Wei-Fu Ji, ${ }^{b}$ Kuei-Sen Chang, ${ }^{c}$ Kuei-Ting Hsu, ${ }^{d}$ Jui-Ming Yeh ${ }^{b}$ \\ and Wei-Ren Liu (D)*a
}

In this study, we fabricated a novel sandwich structure by combining reduced graphene oxide ( $\mathrm{rGO}$ ), polyvinylidene difluoride (PVDF) and polyurethanes (PU) to construct a composite film for anti-corrosion applications. The corrosion performances of the rGO/PVDF/PU multi-layered coatings on carbon steel were evaluated in $30 \mathrm{wt} \%$ boiling sulfuric acid solution for $1 \mathrm{~h}$ and $3.5 \mathrm{wt} \% \mathrm{NaCl}$ electrolyte for electrochemical tests. The results show that the addition of PU and rGO dramatically improves the anticorrosion performance of the bare PVDF coating and the optimized content of $\mathrm{rGO}$ is $7 \mathrm{wt} \%$. The anticorrosion performances of corrosion potential, corrosion current and protection efficiency after protection coating are measured to be $-757 \mathrm{mV}, 0.1 \mu \mathrm{A} \mathrm{cm} \mathrm{cm}^{-2}$ and $99 \%$, respectively. The excellent results obtained could be explained by barrier effects, where the hydrophobic property and high-aspectratio of $r G O$ in the PVDF matrix inhibit corrosion factors from diffusing into the substrate.

Received 19th May 2017

Accepted 18th June 2017

DOI: $10.1039 / \mathrm{c} 7 \mathrm{ra0} 5674 \mathrm{e}$

rsc.li/rsc-advances

strong van der Waals interaction and the out-of-plane $\pi$ bond

\section{Introduction}

Corrosion protection is very important technology in the metallic finishing industry. Corrosion is a very costly problem, which has a major impact on the economies of industrial nations. However, corrosion cannot be fully inhibited. Thus, corrosion control strategies focus on slowing the kinetics and/ or altering its mechanism. Nowadays, there are different strategies for corrosion protection, including cathodic protection, ${ }^{1,2}$ protective coatings, ${ }^{3-5}$ corrosion inhibitors ${ }^{6,7}$ and other. ${ }^{8-10}$ Recently, graphene has emerged as a promising building block in polymer matrices on account of its unique geometry and novel physical properties, such as high specific surface area, superior mechanical strength, high aspect ratio, electrical mobility, thermal conductivity and barrier properties. ${ }^{11-18}$ As reported in the literature, graphene has great application potential in the corrosion protection field. Additionally, there is increasing research interest in related materials, such as GO and rGO. ${ }^{19} \mathrm{GO}$ is the exfoliated product of graphite oxide, which is prepared via a modified Hummers method from graphite power. ${ }^{20}$ Subsequently, GO can be transformed into rGO via chemical, ${ }^{21,22}$ thermal, ${ }^{23,24}$ or electrochemical reduction. ${ }^{25}$ However, one major challenge in the preparation of graphene is its irreversible agglomeration and restacking as a result of

\footnotetext{
${ }^{a}$ Department of Chemical Engineering at Chung Yuan Christian University (CYCU), Chung Li 32023, Taiwan, Republic of China. E-mail: WRLiu1203@gmail.com ${ }^{b}$ Department of Chemistry at CYCU, Chung Li 32023, Taiwan, Republic of China ${ }^{c}$ Refining and Manufacturing Research Institute at CPC Corporation, Chiayi City 60051, Taiwan, Republic of China

${ }^{d}$ Department of Chemical Engineering, Army Academy, Taoyuan City 32092, Taiwan, Republic of China
} between the individual graphene nanosheets. On the other hand, after the oxidation reaction, oxygen-containing functional groups such as epoxy, hydroxyl, carboxyl and carbonyl groups are located either on the basal plane or on the edge of the single atom thin sheet. The presence of these functional groups causes the GO sheets to be strongly hydrophilic, which allows GO to swell and disperse in various polar solvents readily. ${ }^{26}$ Chang et al. reported that graphene provides more desirable anti-corrosion properties for polyaniline than clay due to its higher aspect ratio. ${ }^{27}$ They reported that the well-dispersed graphene displayed remarkable anti-corrosion properties because of enhanced barrier properties. ${ }^{28}$ Unfortunately, the presence of strong intrinsic van der Waals attraction between the fillers and the absence of bonding between graphene and the polymer matrix pose a substantial obstacle to obtain graphene-based composites with excellent dispersion and compatibility. To a great extent, this impairs the anti-corrosion performance of graphene-based composites. Meanwhile, nanocomposites synthesized containing graphene-based materials such as graphene nanoplates (GNPs), graphene nanosheets (GNSs) and GO display excellent beneficial properties when employed in diverse applications. ${ }^{29-35}$ Research associated with conductive graphene, which has a relatively high aspect ratio of $\sim 500$, has attracted much interest. ${ }^{36}$ The lower density and higher aspect ratio of conductive graphene compared with that of non-conductive clay platelets triggered its potential application as an advanced gas barrier polymer nanocomposite. ${ }^{27}$ Mo et al. reported that functionalized graphene (FG) and functionalized graphene oxide (FGO) were obtained by treating graphene and GO with 3- 
aminopropyltriethoxysilane (APTES). ${ }^{37}$ Then, they fabricated a series of PU-based composite coatings reinforced with well dispersed FG and different surface texture densities in the PU matrix which displayed anti-corrosion properties. Singh et al. proved that GO polymer composite coatings are an effective protective shield against the oxidation and corrosion of metal. ${ }^{38}$ Zhang et al. reported a PVDF/GO solution via a simple blending method. ${ }^{25}$ The oxygen containing groups in GO platelets allow them to be dispersed in PVDF solution homogeneously and prevent agglomeration, and the GO can be translated to RGO via thermal reduction during the hot-molding process. The enhanced absorption and dielectric property of PVDF/rGO composites were also investigated. Karan et al. demonstrated that the Fe-rGO/PVDF nanocomposite exhibits superior piezoelectric energy harvesting and ferroelectric properties, where a higher proportion of the polar piezoelectric $\gamma$-phase of PVDF is achieved by simply using the solvent casting method, which exclusively produced an output voltage of $5.1 \mathrm{~V}$ and short circuit current of $0.254 \mu \mathrm{A}$ without the application of electrical poling. ${ }^{41}$

In our previous studies, we demonstrated that the corrosion protection properties of nano-graphene/epoxy composites coated on metal surfaces are capable of enhancing the corrosion protection of pure metals effectively. ${ }^{39,40}$ In this study, we further fabricate anticorrosive $\mathrm{rGO} / \mathrm{PVDF} / \mathrm{PU}$ coatings which provide two-fold protection against metal corrosion. In the first step of the protection mechanism, the hydrophobic graphene is proven to display excellent corrosion protection capability. Second, the well-dispersed reduced graphene nanosheets in the PVDF matrix increase the tortuosity of the diffusion pathway of $\mathrm{H}_{2} \mathrm{O}$ and $\mathrm{O}_{2}$ molecules. The detailed anticorrosion performance of the GPN coated on cold-rolled steel (CRS) is evaluated through a series of electrochemical corrosion measurements in a corrosive medium.

\section{Experimental}

\subsection{Sample preparation}

Polyvinylidene difluoride (Kureha, KF9200), N-methyl-2pyrrolidone (Alfa), sodium nitrate (J. T. Baker), potassium permanganate (SHOWA) and hydrogen peroxide (J. T. Baker) were used as received. Aqueous sulfuric acid and hydrochloric acid were purchased from Echo Chemical Co. Ltd. Graphene nanosheets (SFG44-GNS) prepared from SFG44 synthetic graphite powder (TIMCAL®) were used. The detailed experimental conditions are reported in our previous work. ${ }^{20}$ The obtained graphene oxide powder was subjected to thermal exfoliation at $1050{ }^{\circ} \mathrm{C}$ in air for $30 \mathrm{~s}$. This procedure resulted in few-layer reduced graphene nanosheets. The GNSs were characterized via SEM, X-ray diffraction, Raman spectroscopy, and TEM. A series of rGO/PVDF nanocomposite coatings with $0 \mathrm{wt} \%, 1 \mathrm{wt} \%$ and $7 \mathrm{wt} \%$ GNSs in PVDF (denoted as GPN, GPN1 and GPN7, respectively) were fabricated as follows: first, $4 \mathrm{~g}$ PVDF was dissolved in $20 \mathrm{~g}$ NMP. Then $0.043 \mathrm{~g}$ rGO was mixed using a homogenizer for $40 \mathrm{~min}$ at room temperature. After mixing, drops of the mixture solution were spread on a stainless steel plate followed by the dipping coating process. Finally, cured GPN membranes were obtained with a thickness of $c a$.

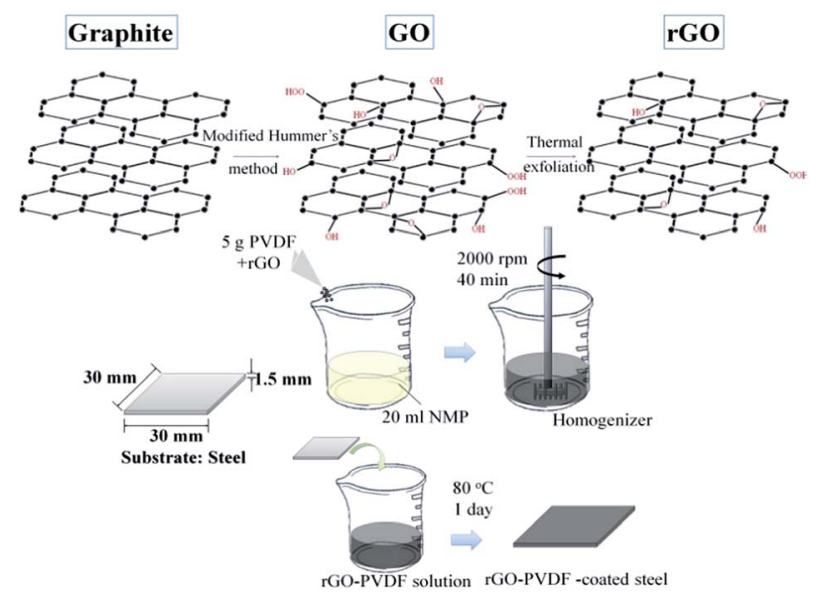

Fig. 1 Preparation of the rGO-PVDF composite film.

$120 \mu \mathrm{m}$ at $60{ }^{\circ} \mathrm{C}$ overnight in a vacuum oven. Graphene/PVDF nanocomposite coatings with different mass fractions of reduced GNSs were prepared in the same way. The fabrication of the GPNs is illustrated schematically in Fig. 1. The whole process of three-layer coating is described as follow: the PU coating was used as the first coating layer on a steel substrate via the dipping coating method. After drying at ambient temperature for one day, the second layer, PVDF, was spread on the asprepared substrate by dipping coating. Then it was placed in an oven at $60{ }^{\circ} \mathrm{C}$ overnight. Finally, the substrate was coated with the $\mathrm{rGO} / \mathrm{PVDF}$ nanocomposite in the same way as the previous step. Thus, the multilayer protecting structure of RGO/PVDF/PU was obtained on the steel substrate.

\subsection{Electrochemical corrosion studies}

Electrochemical corrosion measurement was performed on a VoltaLab 50. All electrochemical corrosion measurements were performed in a double-wall jacketed cell covered with a glass plate, in which water was maintained at a constant operational temperature of $25 \pm 0.5{ }^{\circ} \mathrm{C}$. The open-circuit potential (OCP) at the equilibrium state of the system was recorded as the corrosion potential $\left(E_{\text {corr }}\right.$ in $\mathrm{mV}$ versus the saturated calomel electrode (SCE)). Tafel plots were obtained by scanning the potential from -500 to $500 \mathrm{mV}$ above $E_{\text {corr }}$ at a scan rate of $10 \mathrm{mV} \mathrm{min}^{-1}$. The corrosion current $\left(I_{\text {corr }}\right)$ was determined by superimposing a straight line along the linear portion of the cathodic or anodic curve and extrapolating it through $E_{\text {corr }}$. The corrosion rate $\left(R_{\text {corr }}\right.$, in milli-inches per year, MPY) was calculated using eqn (1):

$$
E_{\text {corr }}(\mathrm{MPY})=\left[0.13 I_{\text {corr }}(\text { E.W. })\right] /[A \times d]
$$

where, E.W. is the equivalent weight (g per eq.), $A$ is the area $\left(\mathrm{cm}^{2}\right)$, and $d$ is the density $\left(\mathrm{g} \mathrm{cm}^{-3}\right)$. An AutoLab (PGSTAT302N) potentiostat/galvanostat was employed to perform AC impedance spectroscopy measurements. Impedance measurements were carried out in the frequency range of $100 \mathrm{kHz}$ to $100 \mathrm{MHz}$ with pure iron (area $1 \times 1 \mathrm{~cm}^{2}$ ) as the working electrode embedded in epoxy, Pt as the counter electrode, and SCE as the 
reference electrode. The working electrode was first maintained in the test environment for $40 \mathrm{~min}$ before the impedance run. All experiments were performed at room temperature. All raw data were repeated at least three times to ensure reproducibility and statistical significance.

\section{Results and discussion}

The SEM image in Fig. 2(a) shows that the synthesized rGO are flexible flakes with a rough morphology and thickness in the nanoscale. The thickness of the rGO after thermal treatment was smaller than that of the GO flakes. The decrease in thickness of the rGO nanosheets is due to several factors, including the removal of oxygen-containing functional groups by heating and elimination of the interlamellar water present in graphite oxide which depends on the relative humidity. The TEM images reveal that the synthesized rGO is transparent and very flexible (Fig. 2(b)). Furthermore, there were some folds in the flexible sheets, which indicate that the rGO was very thin. Since all the synthesized materials are nanostructured, embedding them in the PVDF matrix as fillers is likely to enhance the anticorrosion performance of PVDF. Due to the presence of fillers in the coating matrix, rGO serves as a barrier, which enhances the coating impermeability by increasing the tortuosity of the diffusion pathway of molecules in the coating (physical barrier effect). Generally, fillers with a high aspect ratio and large fillermatrix interfacial area are beneficial for increasing the tortuosity of the diffusion pathway. ${ }^{27}$ The flake-like GPNs are excellent anti-corrosion fillers due to the following reasons. On one hand, the aspect ratio of GPNs is high because the lateral dimension of GPNs is micron-sized. ${ }^{42}$

The XRD patterns of the graphite, GO and rGO are shown in Fig. 2(c). As shown in Fig. 2(c-2), GO has an intense crystalline peak at $10.5^{\circ}$, which corresponds to a $d$-spacing of $8.42 \AA$. The
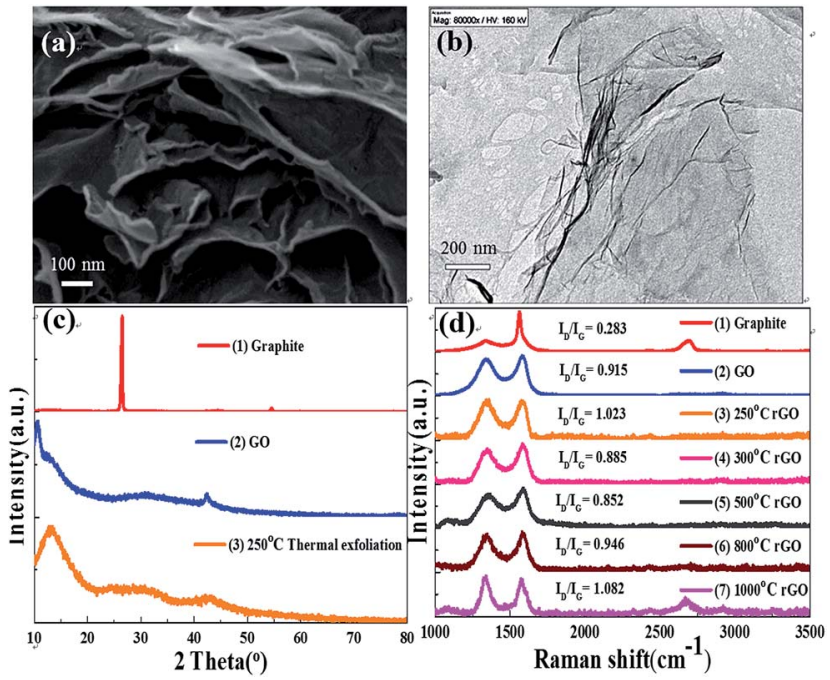

Fig. 2 Characterization of graphite, GO and rGO. (a) and (b) SEM and TEM images of $r G O$, respectively. (c) XRD patterns of graphite, GO and rGO and (d) Raman spectra of graphite, GO and reduced GO obtained at different reducing temperatures. interlayer spacing of the GO sheets is attributed to the oxygenated functional groups introduced by the modified Hummers method. The XRD pattern of rGO is shown in Fig. 2(c-3), in which the peak of GO is weakened. The rGO peak at $13.4^{\circ}$ corresponds to the $d$-spacing of $6.6 \AA$, which is less than that of GO due to the emission of oxygen-containing functional groups. ${ }^{43}$ The results of the $d$-spacing demonstrate that the reduction was successful. Raman spectroscopy was implemented to characterize the structure of the synthesized materials. Fig. 2(d) shows the Raman spectra of graphite, GO and rGO. rGO displays a D band at $1350 \mathrm{~cm}^{-1}$ corresponding to the vibration of graphene defects or edges and a $\mathrm{G}$ band at 1598 $\mathrm{cm}^{-1}$ which is assigned to the in-phase vibration of the graphite lattice.

Cross-section SEM was performed to observe the structure of the multiple anti-corrosion layers. Fig. 3(a) shows that there were good connections between the layers and the substrate. The pure PU layer presented in Fig. 3(a) exhibits a smooth and uniform fracture surface without obvious defects, which could also be a great attachment to non-organic steel substrates. On the other hand, when a small amount of rGO was added into the PVDF matrix, a rougher surface and some cracks appeared which were initiated by the interfacial interaction between rGO and the PVDF matrix. Moreover, the PVDF in the middle of the structure acted a thermal buffer layer, reduced to determine graphene dispersed situation in the PVDF matrix and enabled to descript the degree of exfoliation. Fig. 3(b) shows the TEM micrograph of the PVDF composite of graphene with the graphene content of $7 \mathrm{wt} \%$. The gray region of the photograph represents the domain of PVDF matrix and the black lines correspond to the cross section of graphene nanosheets. This indicates that the attachment of carboxylic groups onto the graphene surface effectively enhances the compatibility between reduced-graphene nanosheets and the polymer matrix, which leads to improved dispersion. In addition, the number of graphene layers is less than 10 and the folds appear. The reduced-graphene nanosheets could be described as few-layer graphene.

Graphene is a flat monolayer of carbon atoms tightly packed into a two-dimensional (2D) honeycomb lattice. However, during the reduction process, graphene oxide can easily aggregate into graphite again. In order to prevent this for occurring, we had to optimize the contents of the co-doped polymer slurry.

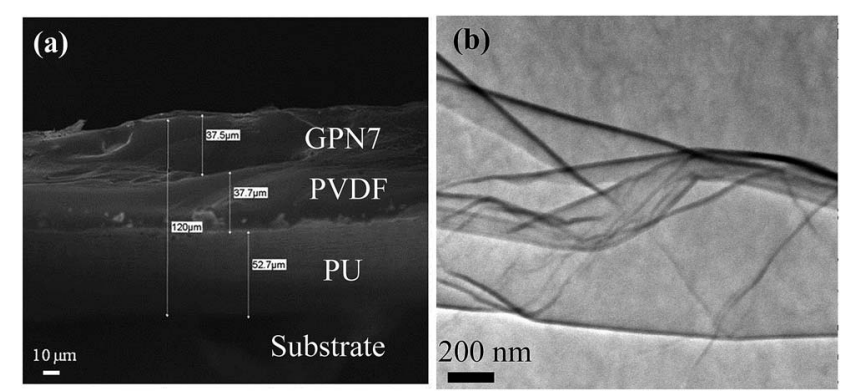

Fig. 3 (a) Cross-section image of the PU/PVDF/rGO-PVDF multiple layers and (b) TEM image of GPN7. 
GPN blended with different contents $(0,3,5,7,10$ and $13 \mathrm{wt} \%)$ of reduced-graphene oxide was boiled in sulfuric acid for $1 \mathrm{~h}$. We measured the weight difference in grams of the samples and the $0 \mathrm{wt} \%$ sample and defined the weight difference per unit area $\left(\mathrm{m}^{2}\right)$ as the weight loss. Fig. 4 shows that the best anticorrosion performance was observed for GPN7. If the concentration was less than $7 \mathrm{wt} \%$, the amount of graphene was not strong enough to defend the corrosive medium. Sulfuric acid molecules diffuse into the depth of the substrate to destroy the steel easily. On the contrary, when the concentration was over 7 $w t \%$, the probability of aggregation between graphene layers increases. To avoid the aggregation in the slurry, we selected a good dispersion agent for graphene, NMP. NMP is not only a powerful solvent for dispersing SWCNTs, ${ }^{44,45}$ graphene, ${ }^{46}$ and GO, ${ }^{47}$ but also a solvent to dissolve PVDF polymer. In addition, we used the sonication method for $30 \mathrm{~min}$ and the assistance of a homogenizer to overcome the aggregation problem. The greater anti-corrosion performance evident in GPN7 was also further analyzed. The inset in Fig. 4 displays the SEM image of the composition optimized $\mathrm{rGO} / \mathrm{PVDF} / \mathrm{PU}$ sample after acid corrosion tests. Compare to the fresh coating shown in Fig. 3(a), the structure of GPN7 changed from a dense structure to a porous structure. In comparison, the PVDF and PU layers remained dense even after heavy corrosion tests in $30 \mathrm{wt} \%$ boiling sulfuric acid solution for $1 \mathrm{~h}$. The results confirm that the three-layer sandwich structure of $\mathrm{rGO} / \mathrm{PVDF} / \mathrm{PU}$ efficiently protects the substrate from acid corrosion.

It is generally believed that a higher contact angle value denotes a more hydrophobic surface. ${ }^{48}$ The above results indicate that the surface hydrophobic property depends on the presence of the functionalized groups containing carbonoxygen bonds in graphene. The enhancement of the hydrophobic property prevents the absorption of water through the coatings. Fig. 5(a) shows the change in the water contact angle with the addition of rGO at different reduction temperatures under Ar. As the reduction temperature increases, the more functionalized groups containing oxygen vaporize into $\mathrm{CO}_{2}$,

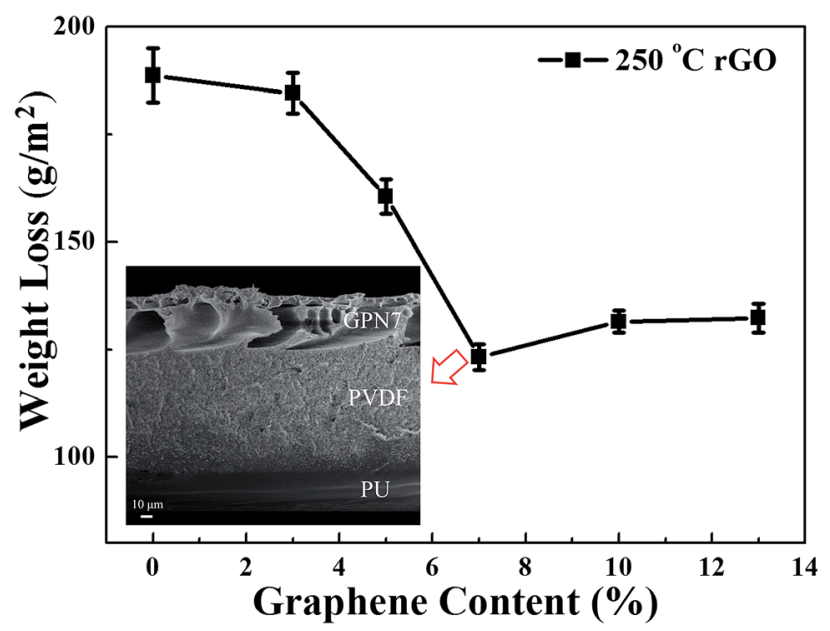

Fig. 4 Mass loss of composite membranes with different contents of rGO, PVDF and rGO-PVDF. Inset: SEM image of the sample after the corrosion tests.
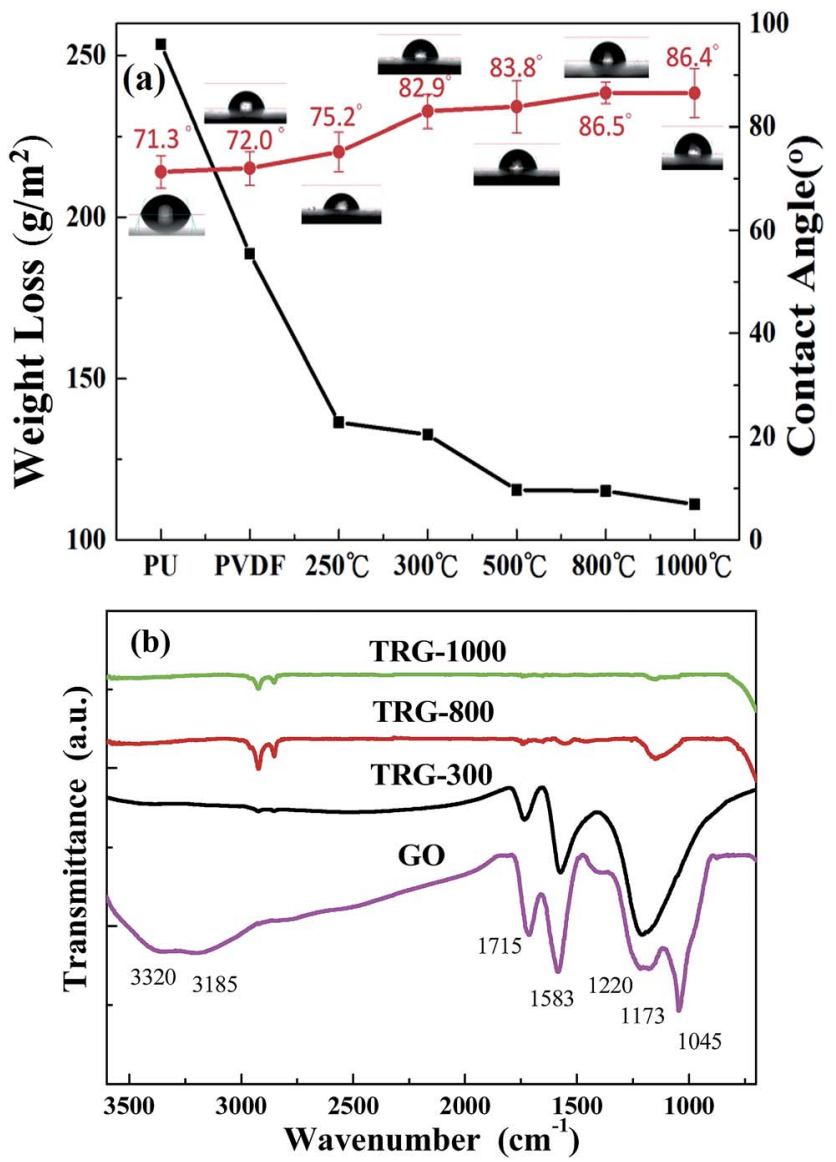

Fig. 5 (a) Water contact angle and weight loss of the composite films of $7 \mathrm{wt} \% \mathrm{rGO}-\mathrm{PVDF}$ with different reduction temperatures in the anticorrosion test in $30 \mathrm{wt} \% \mathrm{H}_{2} \mathrm{SO}_{4}$ for $1 \mathrm{~h}$ and (b) FITR of $\mathrm{GO}$ obtained at different reduction temperatures.

which causes the graphene to become hydrophobic and enhances the blocking capacity of the coating. The contact angle of a water droplet on the sample surface was increased from $\sim 72^{\circ}$ for the PVDF surface to $\sim 86.4^{\circ}$ for GPN7s by tuning the reduction temperature of graphene. The water repellent ability of the GPN coatings is a very important parameter, which was simultaneously determined during the sulfuric test. The result indicates that the weight loss decreased from 188.7 to $115.2 \mathrm{~g} \mathrm{~m}^{-2}$ which improved the anti-corrosion efficiency by about 39\%. The higher reduction temperature resulted in a better hydrophobic phenomenon. Fig. 5(b) shows FITR spectra of GO under different reduction temperatures. Obviously, with an increase in reduction temperature from $300{ }^{\circ} \mathrm{C}$, $800{ }^{\circ} \mathrm{C}$ to $1000{ }^{\circ} \mathrm{C}$, the corresponding oxygen-containing functional groups decreased. Thus, the surface properties of the GPN layer could be easily tuned by the reduction temperature.

Fig. 6(a) shows the PU, PVDF and GPN7 composite coatings, where the left three samples displayed resistance to the sulfuric acid. According to the test, although GPN7 had the best anticorrosion performance, there were some defects on the edges of the steel substrates. The GPN7 membrane peeled off from the substrate because of the weak interaction. On the other hand, the PU coating was still intact and in good shape as determined 

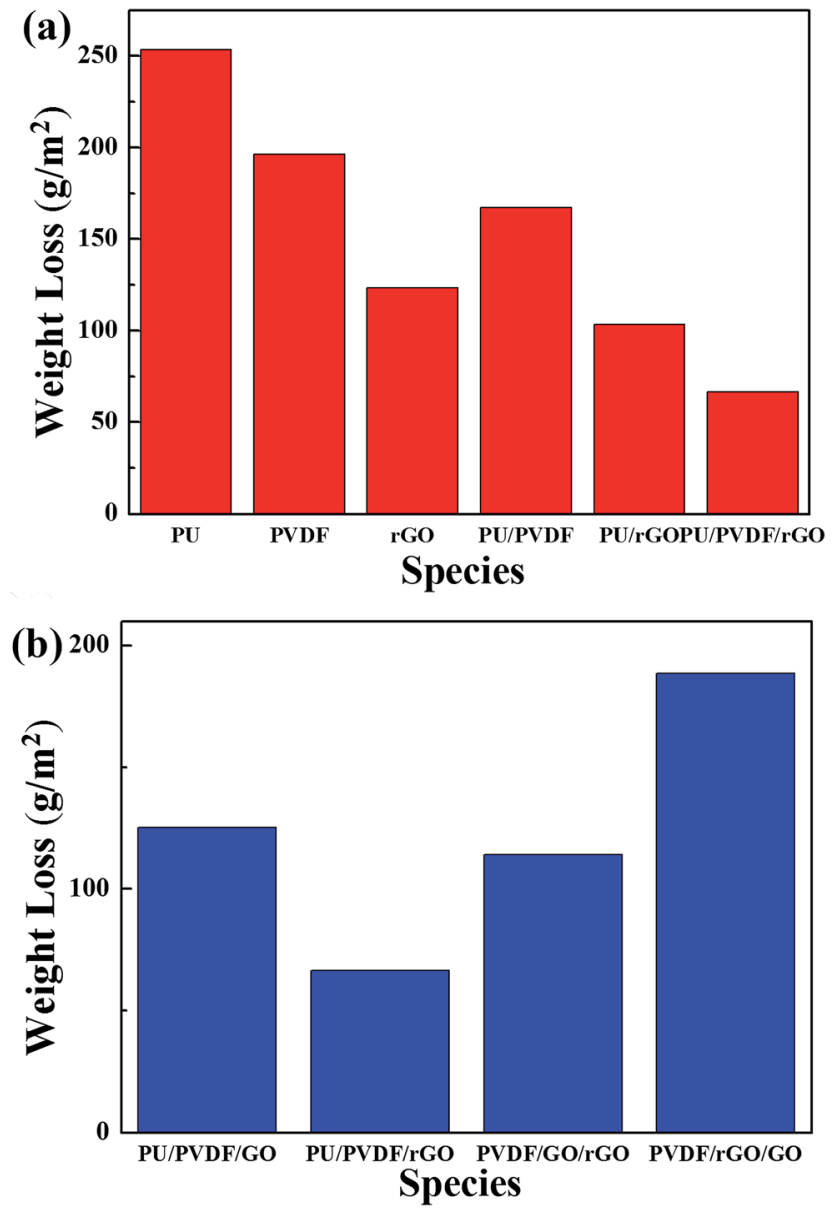

Fig. 6 (a) Anti-corrosion properties of each composite membrane and the structured layers and (b) anti-corrosion properties of the sandwich structure layers by arrangement.

from is physical properties. There were no obvious cracks on its surface structure, but its chemical stability was not as good as GPN7. On the basis of the corrosion test, we combined the advantages of PU and GPN7 and built a new structure of two layers with the same thickness. Fig. 6(a) demonstrates that by using the structured technique, the anti-corrosion performance improved evidently. In order to overcome the high temperature system, the structure must endure thermal expansion tension. We integrated the three membranes and built composite sandwich structures to determine the optimization permutation, and the results are shown in Fig. 6(b). We used two types of additives, GO and rGO as obstacles in the PVDF matrix. It is obviously seen that there were good blocking effects when the GPN7 membrane was on the outside. Moreover, it also had a good performance because the PU polymer played the role of a primer. From the physical properties of the sandwich structures we successfully determined the optimal sandwich structure.

On the basis of a series of electrochemical measurements (i.e., corrosion potential, polarization resistance, and corrosion current measured in a corrosive medium) we concluded that the GPN7 coating is superior at protecting the CRS electrode against corrosion than the common PU and PVDF coatings. The Tafel polarization curves of the bare substrate, pure PVDF, GPN1 and GPN7 composite coatings exposed to $3.5 \mathrm{wt} \% \mathrm{NaCl}$ solution are displayed in Fig. 7. Information about the corrosion current can be obtained by extrapolating the Tafel plots from both the cathodic and anodic polarization curves for the respective corrosion processes. ${ }^{\mathbf{4 9 , 5 0}}$ Extrapolating the cathodic and anodic polarization curves to their point of intersection provides both the corrosion potential $\left(E_{\text {corr }}\right)$ and the corrosion current $\left(I_{\text {corr }}\right)$. In general, a lower $I_{\text {corr }}$ value represents a lower corrosion dynamic rate. Corrosion protection studies were performed on samples with $10 \pm 1 \mu \mathrm{m}$ thick coatings that were immersed in a corrosive medium for $40 \mathrm{~min}$. The protection efficiency $(\eta)$ of the coatings on the substrate was calculated from the $I_{\text {corr }}$ values according to eqn (2): ${ }^{51}$

$$
\eta=\frac{I_{\text {corr }}^{\circ}-I_{\text {corr }}}{I_{\text {corr }}^{\circ}} \times 100 \%
$$

where, $I_{\text {corr }}^{\circ}$ and $I_{\text {corr }}$ are the corrosion current densities of the bare and coated substrate, respectively. The Tafel plots for the sample-coated CRS electrode gave a corrosion potential of $E_{\text {corr }}$ $=-883,-803$ and $-757 \mathrm{mV}$ for the PVDF, GPN1 and GPN7 coatings, respectively, which was more positive than that for the bare CRS electrode, where $E_{\text {corr }}=-924 \mathrm{mV}$. Moreover, the corrosion current of the PVDF, GPN1-coated and GPN7-coated CRS electrodes was $c a .12 .62,0.32$ and $0.10 \mu \mathrm{A} \mathrm{cm}^{-2}$, respectively, which was significantly lower than that of the bare CRS electrode $\left(147.91 \mu \mathrm{A} \mathrm{cm}{ }^{-2}\right)$. The corresponding $I_{\text {corr }}$ decreased considerably when we used the GPN-coated bare CRS electrode with the additive of reduced graphene oxide to produce hydrophobic properties. Moreover, the corrosion potential of the GPN7-coated CRS electrode was more positive than that of the GPN1-coated CRS electrode, as $E_{\text {corr }}$ increased from -803 to $-757 \mathrm{mV}$ ( $v s$. SCE). These electrochemical measurement results show that the GPN7 coating provided better protection against corrosion for the CRS electrode than the other coatings.

Dielectric spectroscopy, which is also known as impedance spectroscopy or electrochemical impedance spectroscopy (EIS),

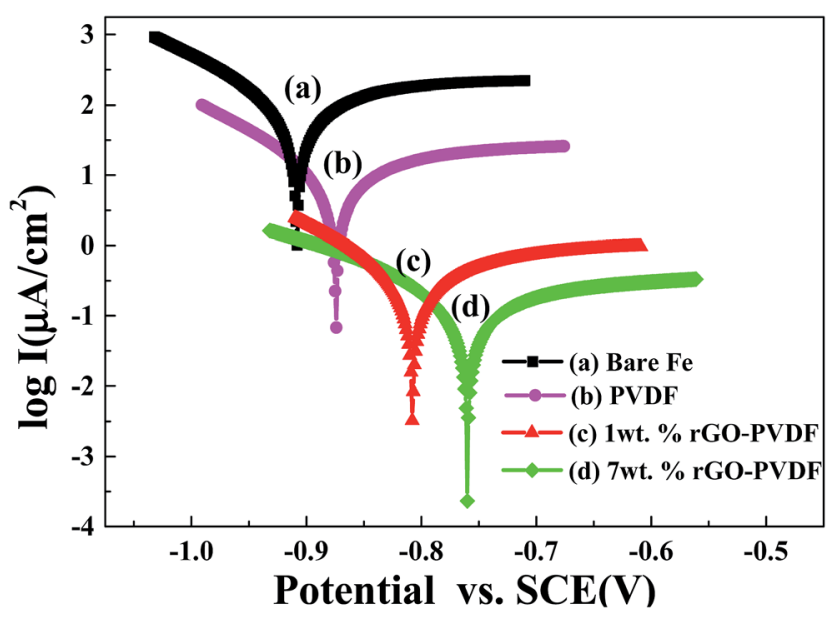

Fig. 7 Tafel plots for (a) bare steel, (b) PVDF-coated, (c) 1 wt\% rGOPVDF coated and (d) 7 wt\% rGO-PVDF coated CRS electrodes measured in $3.5 \mathrm{wt} \% \mathrm{NaCl}$ aqueous solution. 


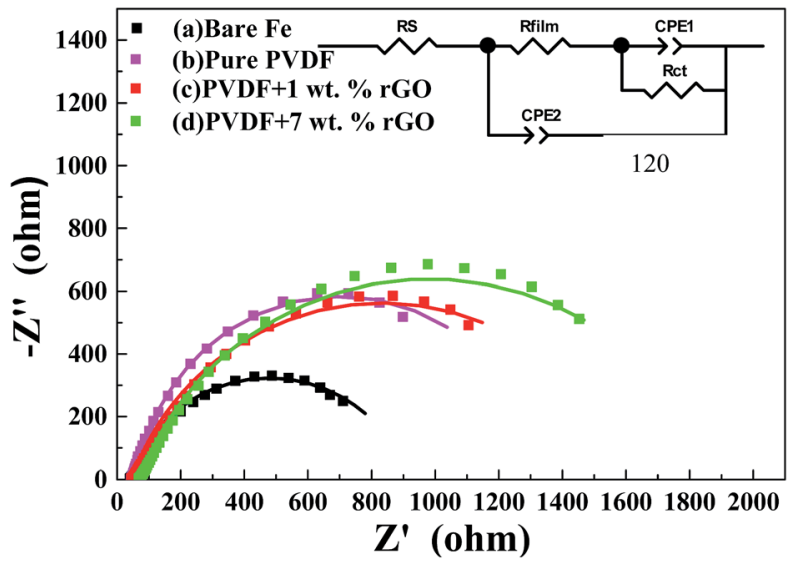

Fig. 8 Nyquist plots of (a) bare steel, (b) PVDF-coated, (c) 1 wt\% rGOPVDF coated, and (d) $7 \mathrm{wt} \% \mathrm{rGO}-\mathrm{PVDF}$ coated CRS electrodes experimental data and-fitted data).

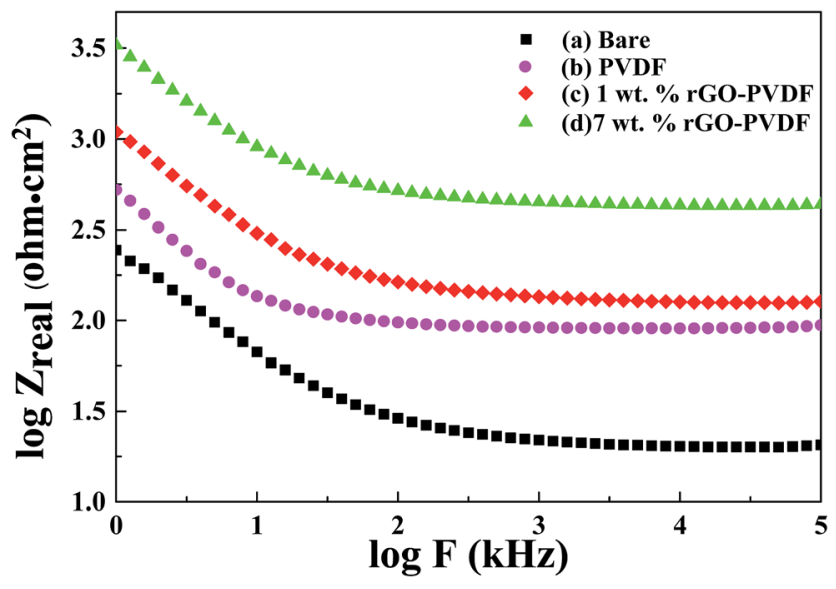

Fig. 9 Bode plots for (a) bare, (b) PVDF-coated, (c) 1 wt\% rGO-PVDF coated, and (d) 7 wt\% rGO-PVDF coated CRS electrodes.

is used to measure the dielectric properties of a medium and express them as a function of frequency. ${ }^{52,53}$ EIS was also used to examine the difference in the activity between the surface of the bare CRS electrode and the PVDF- or GPN-coated electrodes. Impedance is a complex resistance when alternate current flows through a circuit consisting of capacitors or a combination of circuits. ${ }^{54}$ EIS measurements result in currents over a wide range of frequencies. Complex nonlinear least-squares procedures available in numerous EIS-data-fitting computer programs could be used to fit the model to the experimental data in order to obtain the Randles circuit parameters. For the simulation studies, the corrosion of the metal was modeled using an equivalent circuit (called a Randles circuit), as illustrated in Fig. 9, which consists of a double-layer capacitor connected in parallel with a charge-transfer resistor and in series with an electrolyte solution resistor. The impedance $(Z)$ depends on the charge-transfer resistance $\left(R_{\mathrm{ct}}\right)$, the solution resistance $\left(R_{\mathrm{s}}\right)$, the capacitance of the electrical double layer, and the frequency of the AC signal $(\omega)$ as follows:

$$
\begin{aligned}
Z & =Z^{\prime}+\mathrm{j} Z^{\prime \prime} \\
& =R_{\mathrm{s}}+\frac{R_{\mathrm{ct}}}{\left[1+\left(R_{\mathrm{ct}} C_{\mathrm{dl}} \omega\right)^{2}\right]}+\frac{\mathrm{j}\left(R_{\mathrm{ct}}{ }^{2} C_{\mathrm{dl}} \omega\right)}{\left[1+\left(R_{\mathrm{ct}} C_{\mathrm{dl}} \omega\right)^{2}\right]}
\end{aligned}
$$

The fitted data fit well to all the experimental electrochemical data. The high-frequency intercept is equal to the solution resistance and the low-frequency intercept is equal to the sum of the solution and charge transfer resistances. ${ }^{55}$ In general, a larger semicircle diameter (charge transfer resistance) represents a lower corrosion rate. Fig. 8 shows the Nyquist plots of the four measured samples. The first sample (a) is uncoated CRS. The series of samples denoted as (b), (c) and (d) represent CRS coated by PVDF, GPN1 and GPN7, respectively. The charge transfer resistances of all the samples, as determined by subtracting the intersection of the high-frequency end from the low-frequency end of the semicircle arc with the real axis, are 710.2, 898.5, 1105 and $1453 \Omega \mathrm{cm}^{2}$, respectively. The EIS Bode plots (impedance $v s$. frequency) of all the samples are shown in Fig. 9. $Z_{\text {real }}$ is a measure of corrosion resistance. ${ }^{56}$ A low $Z_{\text {real }}$ value could be caused by very high capacitance and/or very low resistance of the coating. ${ }^{57,58}$ Also, the capacitance value is related to the extent to which water penetrates the coating. ${ }^{59}$

In the case of the Bode plots, the value of $Z_{\text {real }}$ at the lowest frequency also represents the corrosion resistance. The Bode magnitude plots for the uncoated CRS and CRS coated by PVDF, GPN1 and GPN7 show $Z_{\text {real }}$ values of 20.37, 94.19, 127.35, and $434.51 \Omega \mathrm{cm}^{2}$, respectively, at the low frequency region. These results clearly demonstrate that the GPN7 coating protects the CRS electrode against corrosion better than the PVDF and GPN1 coatings. The increase in impedance for the GPN7-coated electrode can be attributed to the hydrophobicity and barrier effects of GNSs dispersed in the composites of the coating. The hydrophobicity of GPN7 compared to that of PVDF and GPN1 may result from the low wettability and dispersed graphene in the PVDF matrix which increase the tortuosity of the corrosive media diffusion pathway (lower diffusion length), as shown in Fig. 10. ${ }^{60-63}$

Table 1 lists the $E_{\text {corr }}, I_{\text {corr }}$, thickness and protection efficiency (PEF\%) reported in previous work. The protection efficiency was utilized to evaluate the anticorrosive performance of the coatings using eqn (4).

$$
\text { Protection efficiency }(\%)=\frac{I_{\text {corr }}-I_{\text {corr }}(c)}{I_{\text {corr }}} \times 100
$$

where, $I_{\text {corr }}$ and $I_{\text {corr }}(c)$ are the corrosion current values in the absence and presence of the coating, respectively. This result

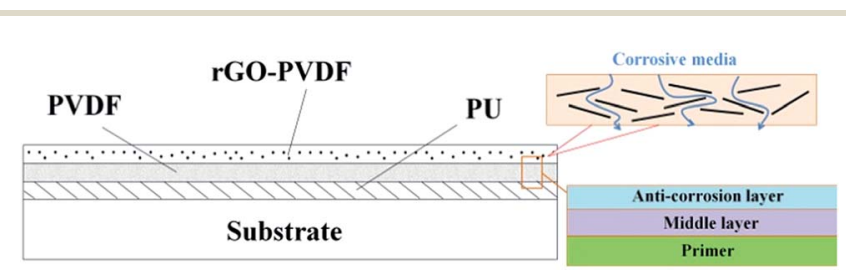

Fig. 10 Schematic representation of the hydrophobic surface and oxygen following a tortuous path through PVDF and RPC materials. 
Table 1 Comparison of the anticorrosive performance, $E_{\text {corr }} I_{\text {corr }}$, thickness and protection efficiency of the electrodes modified with different polymer coatings in saline

\begin{tabular}{|c|c|c|c|c|c|}
\hline Sample & $E_{\text {corr }}^{a}(\mathrm{mV})$ & $I_{\text {corr }}\left(\mu \mathrm{A} \mathrm{cm}^{-2}\right)$ & Protection efficiency (PEF\%) & Thickness $(\mu \mathrm{m})$ & Reference \\
\hline 7 wt $\%$ rGO-PVDF & -757 & 0.1 & $99.9 \%$ & 120 & This work \\
\hline EPGN1 & -432 & 0.15 & $98.9 \%$ & 20 & 28 \\
\hline PAGCs & -537 & 0.38 & $97.4 \%$ & 29 & 27 \\
\hline $\mathrm{PEI} / \mathrm{G}_{1}$ & -10.7 & 0.15 & $99.1 \%$ & 65 & 65 \\
\hline $5 \mathrm{wt} \% \mathrm{RGO} / \mathrm{PANI}$ & -714 & 12.14 & $79.3 \%$ & 50 & 66 \\
\hline
\end{tabular}

indicates that $7 \mathrm{wt} \%$ rGO-PVDF has a better PEF\%, which implies that 7 wt\% rGO-PVDF gave a better anticorrosion protection.

In order to well understand the mechanism of anticorrosion for the rGO-PVDF coatings, the results are attributed to the barrier effects of the materials. Well dispersed graphene layer materials were incorporated into the PVDF coating (i.e. GPN1 and GPN7) which increased the diffusion pathways for reactive chemicals $\left(\mathrm{H}_{2} \mathrm{SO}_{41}\right.$ and $\left.\mathrm{Cl}^{-}\right)$which contributed to a significant enhancement in the corrosion protection of the metallic substrate compared with that of neat PVDF coating. Moreover, GPN7 has a better anticorrosive performance than that of GPN1, with increase in the amount of graphene from $1 \mathrm{wt} \%$ to $7 \mathrm{wt} \%$. This excellent result is attributed to fact that a higher content of graphene added into the polymer coating could provide longer diffusion pathways and promote the enhancement of the corrosion protection of the metallic substrate compared with that of the GPN1 coating, as shown in Fig. 10.

\section{Conclusions}

In this study, we successfully converted graphite into exfoliatedGO via a modified Hummer's method. After thermal reduction by treating the exfoliated-GO with $\mathrm{Ar}$, the reduced graphene nanosheets displayed an improved hydrophobic property to prevent corrosion. As an effective building block, the graphene additive comprehensively enhanced the anti-corrosion properties of the PVDF composite coating due to the obstructive and barrier properties it provided. Also, the optimized additive content was $7 \mathrm{wt} \%$. As the content of rGO was further increased in excess of the optimized additive amount, the anti-corrosion performance of the PVDF composite coatings was weakened. This is because it contributed to a growing number of aggregates across the whole PVDF composite coating and shortened the diffusion path. Finally, we successfully presented a novel PU/PVDF/rGO composite and the sandwich structure demonstrates excellent anti-corrosion effects for metal protection due to its strong mechanical properties.

\section{Acknowledgements}

This research was financially supported by the Ministry of Science and Technology (MOST 105-2622-E-033-003-CC2 and MOST 104-2628-E-033-002-MY3).

\section{Notes and references}

1 G. Shen, Y. Chen and C. Lin, Thin Solid Films, 2005, 489, 130136.

2 H. Yun, J. Li, H. B. Chen and C. J. Lin, Electrochim. Acta, 2007, 52, 6679-6685.

3 B. Wessling, Adv. Mater., 1994, 6, 226-228.

4 P. A. Kilmartin, L. Trier and G. A. Wright, Synth. Met., 2002, 131, 99-109.

5 A. F. Frau, R. B. Pernites and R. C. Advincula, Ind. Eng. Chem. Res., 2010, 49, 9789-9797.

6 M. Quraishi and J. Rawat, Mater. Chem. Phys., 2002, 73, 118122.

7 T. Söylev and M. Richardson, Constr. Build. Mater., 2008, 22, 609-622.

8 K. Kendig, M. Hon and L. Warren, Prog. Org. Coat., 2003, 47, 183-189.

9 A. Kumar, L. Stephenson and J. Murray, Prog. Org. Coat., 2006, 55, 244-253.

10 C. Suryanarayana, K. C. Rao and D. Kumar, Prog. Org. Coat., 2008, 63, 72-78.

11 M. J. Allen, V. C. Tung and R. B. Kaner, Chem. Rev., 2009, 110, 132-145.

12 D. R. Dreyer, R. S. Ruoff and C. W. Bielawski, Angew. Chem., Int. Ed., 2010, 49, 9336-9344.

13 A. K. Geim and K. S. Novoselov, Nat. Mater., 2007, 6, 183-191.

14 K. P. Loh, Q. Bao, P. K. Ang and J. Yang, J. Mater. Chem., 2010, 20, 2277-2289.

15 C. N. R. Rao, A. K. Sood, K. S. Subrahmanyam and A. Govindaraj, Angew. Chem., Int. Ed., 2009, 48, 7752-7777.

16 W. Ma, L. Wu, D. Zhang and S. Wang, Colloid Polym. Sci., 2013, 291, 2765-2773.

17 H. He and C. Gao, ACS Appl. Mater. Interfaces, 2010, 2, 32013210.

18 H. J. Song and N. Li, Appl. Phys. A:, 2011, 105, 827-832.

19 D. Fan, C. Zhang, J. He, R. Hua, Y. Zhang and Y. Yang, J. Mater. Chem., 2012, 22, 18564-18571.

20 W. S. Hummers and R. E. Offeman, J. Am. Chem. Soc., 1958, 80, 1339.

21 D. R. Dreyer, S. Park, C. W. Bielawski and R. S. Ruoff, Chem. Soc. Rev., 2010, 39, 228-240.

22 H. Bai, C. Li and G. Shi, Adv. Mater., 2011, 23, 1089-1115.

23 R. Larciprete, S. Fabris, T. Sun, P. Lacovig, A. Baraldi and S. Lizzit, J. Am. Chem. Soc., 2011, 133, 17315-17321. 
24 Y. Xu, Q. Wu, Y. Sun, H. Bai and G. Shi, ACS Nano, 2010, 4, 7358-7362.

25 X. J. Zhang, G. S. Wang, W. Q. Cao, Y. Z. Wei, M. S. Cao and L. Guo, RSC Adv., 2014, 4, 19594-19601.

26 S. Stankovich, D. A. Dikin, G. H. Dommett, K. M. Kohlhaas, E. J. Zimney, E. A. Stach, S. Stankovich, D. A. Dikin, G. H. Dommett, K. M. Kohlhaas, E. J. Zimney and E. A. Stach, Nature, 2006, 442, 282-286.

27 C. H. Chang, T. C. Huang, C. W. Peng, T. C. Yeh, H. I. Lu, W. I. Hung, C. J. Weng, T. I. Yang and J. M. Yeh, Carbon, 2012, 50, 5044-5051.

28 K. Chang, C. Hsu, H. Lu, W. Ji, C. Chang, W. Li, et al., eXPRESS Polym. Lett., 2014, 8, 243-255.

29 C. Bao, L. Song, C. A. Wilkie, B. Yuan, Y. Guo, Y. Hu, et al., J. Mater. Chem., 2012, 22, 16399-16406.

30 J. R. Potts, O. Shankar, L. Du and R. S. Ruoff, Macromolecules, 2012, 45, 6045-6055.

31 X. Jiang and L. T. Drzal, J. Power Sources, 2012, 218, 297-306. 32 M. Li, X. Huang, C. Wu, H. Xu, P. Jiang and T. Tanaka, J. Mater. Chem., 2012, 22, 23477-23484.

33 B. K. Kim, M. W. Jang, H. C. Park, H. M. Jeong and E. Y. Kim, J. Polym. Sci., Part A: Polym. Chem., 2012, 50, 1418-1423.

34 S. R. Prabakar, Y. H. Hwang, E. G. Bae, D. K. Lee and M. Pyo, Carbon, 2013, 52, 128-136.

35 S. R. Prabakar, Y. H. Hwang, B. Lee, K. S. Sohn and M. Pyo, J. Electrochem. Soc., 2013, 160, A832-A837.

36 Z. Xu and M. J. Buehler, ACS Nano, 2010, 4, 3869-3876.

37 M. Mo, W. Zhao, Z. Chen, Q. Yu, Z. Zeng, X. Wu, et al., RSC Adv., 2015, 5, 56486-56497.

38 B. P. Singh, B. K. Jena, S. Bhattacharjee and L. Besra, Surf. Coat. Technol., 2013, 232, 475-481.

39 Z. Yu, H. Di, Y. Ma, Y. He, L. Liang, L. Lv, et al., Surf. Coat. Technol., 2015, 276, 471-478.

40 Z. Yu, H. Di, Y. Ma, L. Lv, Y. Pan, C. Zhang, et al., Appl. Surf. Sci., 2015, 351, 986-996.

41 S. K. Karan, D. Mandal and B. B. Khatua, Nanoscale, 2015, 7, 10655-10666.

42 H. Wang, Q. Hao, X. Yang, L. Lu and X. Wang, Nanoscale, 2010, 2, 2164-2170.

43 Q. Shao, J. Tang, Y. Lin, J. Li, F. Qin, J. Yuan, et al., J. Power Sources, 2015, 278, 751-759.

44 C. Furtado, U. Kim, H. Gutierrez, L. Pan, E. Dickey and P. C. Eklund, J. Am. Chem. Soc., 2004, 126, 6095-6105.
45 S. Giordani, S. D. Bergin, V. Nicolosi, S. Lebedkin, M. M. Kappes, W. J. Blau, et al., J. Phys. Chem. B, 2006, 110, 15708-15718.

46 Y. Hernandez, V. Nicolosi, M. Lotya, F. M. Blighe, Z. Sun, S. De, et al., Nat. Nanotechnol., 2008, 3, 563-568.

47 J. Paredes, S. Villar-Rodil, A. Martinez-Alonso and J. Tascon, Langmuir, 2008, 24, 10560-10564.

48 C. Zhou, X. Lu, Z. Xin, J. Liu and Y. Zhang, Prog. Org. Coat., 2013, 76, 1178-1183.

49 A. Mitra, Z. Wang, T. Cao, H. Wang, L. Huang and Y. Yan, J. Electrochem. Soc., 2002, 149, B472-B478.

50 D. E. Beving, A. M. McDonnell, W. Yang and Y. Yan, J. Electrochem. Soc., 2006, 153, B325-B329.

51 S. Banerjee, A. Mishra, M. M. Singh, B. Maiti, B. Ray and P. Maiti, RSC Adv., 2011, 1, 199-210.

52 Y. D. Liu, F. F. Fang and H. J. Choi, Langmuir, 2010, 26, 12849-12854.

53 Y. D. Liu, B. J. Park, Y. H. Kim and H. J. Choi, J. Mater. Chem., 2011, 21, 17396-17402.

54 S. M. Park and J. S. Yoo, Anal. Chem., 2003, 75, A455-A461.

55 A. Amirudin and D. Thieny, Prog. Org. Coat., 1995, 26, 1-28.

56 F. Zucchi, V. Grassi, A. Frignani, C. Monticelli and G. Trabanelli, J. Appl. Electrochem., 2006, 36, 195-204.

57 M. B. Kannan, D. Gomes, W. Dietzel and V. Abetz, Surf. Coat. Technol., 2008, 202, 4598-4601.

58 A. S. Hamdy, E. El-Shenawy and T. El-Bitar, Int. J. Electrochem. Sci., 2006, 1, 171-180.

59 G. Reinhard, P. Simon and U. Rammelt, Prog. Org. Coat., 1992, 20, 383-392.

60 I. H. Tseng, Y. F. Liao, J. C. Chiang and M. H. Tsai, Mater. Chem. Phys., 2012, 136, 247-253.

61 J. R. Potts, D. R. Dreyer, C. W. Bielawski and R. S. Ruoff, Polymer, 2011, 52, 5-25.

62 S. Morimune, T. Nishino and T. Goto, ACS Appl. Mater. Interfaces, 2012, 4, 3596-3601.

63 P. Podsiadlo, A. K. Kaushik, E. M. Arruda, A. M. Waas, B. S. Shim, J. Xu, et al., Science, 2007, 318, 80-83.

64 K. C. Chang, W. F. Ji, C. W. Li, C. H. Chang, T. L. Chuang, J. M. Yeh and W. R. Liu, eXPRESS Polym. Lett., 2014, 8(12), 908-919.

65 H. Alhumade, A. Abdala, A. Yu, A. Elkamel and L. Simon, Can. J. Chem. Eng., 2016, 94(5), 896-904.

66 K. Cai, S. Zuo, S. Luo, C. Yao, W. Liu, J. Ma, H. Mao and Z. Li, RSC Adv., 2016, 6, 95965-95972. 